\title{
Flächentreue $C^{1}$-Abbildungen sind Isometrien
}

\author{
Peter Schöpf
}

\begin{abstract}
Peter Schöpf wurde 1946 in Meran geboren. Er studierte in Graz, zuerst theoretische Physik, später reine Mathematik. Neben der Beschäftigung mit Ungleichungen und Problemen der Konvexgeometrie gilt sein Interesse Begründungsfragen für Geometrien und damit zusammenhängend natürlich der Geschichte und den Grundlagen der Mathematik. Gerne lässt er sich ab und zu durch seine Hobbies ablenken - zum Vorteil für sein inneres Gleichgewicht - , und spielt Jazzgitarre oder beschäftigt sich mit Literatur.
\end{abstract}

J. Firey zeigte in [1], daß eine lineare Abbildung $\varphi: \mathbb{R}^{n} \rightarrow \mathbb{R}^{n}$, die für ein $k$ zwischen 1 und $n-1$ das $k$-dimensionale Volumen invariant läßt, eine Isometrie des $\mathbb{R}^{n}$ ist. Im Anschluß daran bewies P. McMullen in [2], daß ein Homöomorphismus $\Phi: \mathbb{R}^{n} \rightarrow$ $\mathbb{R}^{n}$, der das $(n-1)$-dimensionale Volumen (im Sinne von Minkowski) invariant läßt, eine Isomtrie ist. Die analoge Aussage für $2 \leq k \leq n-2$ war leider nicht mit der selben Beweismethode, die mehrmals die isoperimetrische Eigenschaft der Kugel benützt, zu erledigen. Mit Hilfe eines neuen Beweises des Ergebnisses von Firey gelingt es die bisher offengebliebenen Fälle unter der modifizierten Annahme, daß $\Phi$ eine stetig differenzierbare flächentreue Abbildung ist, zu behandeln. Das $k$-dimesionale Volumen werde dabei im Sinne der Differentialgeometrie verstanden.

Satz 1 Es seien $k, n \in \mathbb{N}$ mit $1 \leq k \leq n-1$. Jede lineare Abbildung $\varphi: \mathbb{R}^{n} \rightarrow \mathbb{R}^{n}$, die das $k$-dimensionale Volumen aller $k$-dimensionalen Kugeln im $\mathbb{R}^{n}$ invariant läßt, ist eine Isometrie.

Es gibt - wie man weiss - keine längentreuen, d.h. isometrischen Abbildungen der Kugeloberfläche in die Ebene, aber flächentreue Abbildungen existieren, flächentreue Karten der Erdkugeloberfläche zum Beispiel sind wohlbekannt. Die Situation gibt zu einer ganzen Reihe von Fragen Anlass. Eine davon ist diese: Gegeben sei eine stetig differenzierbare Selbstabbildung des $n$-dimensionalen reellen Raumes $\mathbb{R}^{n}$, welche für ein $k$ mit $1 \leq k \leq n-1$ das Volumen aller $k$-dimensionalen Kugeln invariant lässt. Ist die Abbildung isometrisch? Der vorliegende Beitrag von Peter Schöpf gibt die Antwort. 
Beweis: Zunächst ist $\varphi$ injektiv. Zu einem Vektor $\mathbf{x}_{1} \in \operatorname{ker}(\varphi) \backslash\{\mathbf{o}\}$ gäbe es nämlich weitere Vektoren $\mathbf{x}_{2}, \ldots, \mathbf{x}_{k}$, so daß das von $\mathbf{x}_{1}, \ldots, \mathbf{x}_{k}$ aufgespannte Parallelepiped $k$ dimensionales Volumen ungleich Null hätte, während das Bildepiped $k$-dimensionalen Inhalt Null hätte, im Widerspruch zur Voraussetzung. Wegen $n \in \mathbb{N}$ ist $\varphi$ sogar bijektiv. Es sei nun $B:=\left\{\mathbf{x} \in \mathbb{R}^{n} \mid \quad\|\mathbf{x}\| \leq 1\right\}$ die euklidische Einheitsvollkugel im $\mathbb{R}^{n}$, dann ist $E:=\varphi(B)$ ein $n$-dimensionales Ellipsoid. Wir zeigen, daß alle Hauptachsen gleich lang sind. Sei $Y:=\left\{\mathbf{y}_{1}, \ldots, \mathbf{y}_{n}\right\}$ eine Menge von (paarweise orthogonalen) Hauptachsenvektoren von $E$. Um zu zeigen, daß $\left\|\mathbf{y}_{i}\right\|=\left\|\mathbf{y}_{j}\right\|$ ist, bilden wir mit $k-1$ Vektoren $\mathbf{z}_{2}, \ldots, \mathbf{z}_{k} \in Y \backslash\left\{\mathbf{y}_{i}, \mathbf{y}_{j}\right\}$ die durch $\mathbf{y}_{i}, \mathbf{z}_{2}, \ldots, \mathbf{z}_{k}$ bzw. $\mathbf{y}_{j}, \mathbf{z}_{2}, \ldots, \mathbf{z}_{k}$ aufgespannten Unterräume $U_{i}$ bzw. $U_{j}$. Die Durchschnitte $E \cap U_{i}$ und $E \cap U_{j}$ sind $k$-dimensionale Ellipsoide, deren Urbilder unter $\varphi k$-dimensionale Einheitskugeln sind und mithin dasselbe $k$-dimensionale Volumen besitzen. Das heißt aber, daß $\left\|\mathbf{y}_{i}\right\|=\left\|\mathbf{y}_{j}\right\|$. Somit ist $E=B$ und $\varphi$ ist eine Isometrie des $\mathbb{R}^{n}$.

Bemerkung: Läßt $\varphi$ die $k$-dimensionalen Volumina aller $k$-dimensionalen Simplices invariant, dann zieht man bekannte Sätze über die Approximation der $k$-dimensionalen Kugel durch disjunkte Vereinigungen $k$-dimensionaler Simplices heran, um die Invarianz des entsprechenden Kugelvolumens zu zeigen. Mit Satz 1 gewinnt man so erneut die Behauptung von Firey.

Im folgenden bezeichne $\operatorname{Vol}_{k}(M)$ das $k$-dimensionale Volumen der Menge $M \subset \mathbb{R}^{n}$. Speziell sei $\kappa_{k}$ das Volumen der $k$-dimensionalen Einheitskugel.

Satz 2 Es seien $k, n$ wie in Satz 1. Ist $\Phi: \mathbb{R}^{n} \rightarrow \mathbb{R}^{n}$ eine einmal stetig differenzierbare Abbildung, die das $k$-dimensionale Volumen aller $k$-dimensionalen Kugeln im $\mathbb{R}^{n}$ invariant läßt, dann ist $\Phi_{\mathbf{x}}^{\prime}$ für alle $\mathbf{x} \in \mathbb{R}^{n}$ eine Isometrie.

Beweis: Angenommen es gibt ein $\mathbf{a} \in \mathbb{R}^{n}$ und $\Phi_{\mathbf{a}}^{\prime}$ ist keine Isometrie, dann bildet $\Phi_{\mathbf{a}}^{\prime}$ die Einheitsvollkugel $B$ des $\mathbb{R}^{n}$ auf ein Ellipsoid $E$ mit $E \neq B$ ab. Es sei nun $Y:=\left\{\mathbf{y}_{1}, \ldots, \mathbf{y}_{n}\right\}$ wieder eine Menge von Hauptachsenvektoren von $E$, wobei jetzt aber auch Nullvektoren vorkommen können. Da $E \neq B$ ist, gibt es $k$ Vektoren $\mathbf{z}_{1}, \ldots, \mathbf{z}_{k} \in$ $Y$, die als Hauptachsenvektoren genommen ein Teilellipsoid $E^{*} \subset E$ aufspannen mit $\operatorname{Vol}_{k}\left(E^{*}\right) \neq \kappa_{k}$. (Im Extremfall ist $\left.\operatorname{Vol}_{k}\left(E^{*}\right)=0\right)$.

Sei nun $B^{*}$ eine solche $k$-dimensionale Teilkugel von $B$, daß $\Phi_{\mathbf{a}}^{\prime}\left(B^{*}\right)=E^{*}$. Ab nun betrachten wir die Einschränkung von $\Phi$ auf $B^{*}$ als Parametrisierung $\Psi: B^{*} \rightarrow \mathbb{R}^{n}$ der Bildmenge $\Phi\left(B^{*}\right)=\Psi\left(B^{*}\right)$. Für das weitere sei $\mathbf{e}_{1}, \ldots, \mathbf{e}_{k}$ eine Orthonormalbasis des von $B^{*}$ aufgespannten Unterraumes und $\Psi_{\mathbf{a}}^{\prime t}$ die transponierte lineare Abbildung zu $\Psi_{\mathbf{a}}^{\prime}$. Die durch

$$
g(\mathbf{x}):=\operatorname{det}\left(\Psi_{\mathbf{x}}^{\prime t} \circ \Psi_{\mathbf{x}}^{\prime}\right)
$$

definierte Gramsche Determinante $g$ ist stetig in $\mathbf{x}$ und es gilt

$$
\operatorname{Vol}_{k}\left(E^{*}\right)=\operatorname{Vol}_{k}\left(\Psi_{\mathbf{x}}^{\prime}\left(B^{*}\right)\right)=\sqrt{g(\mathbf{x})} \operatorname{Vol}_{k}\left(B^{*}\right)=\sqrt{g(\mathbf{x})} \kappa_{k},
$$

wobei entweder $0 \leq \sqrt{g(\mathbf{a})}<1$ oder $1<\sqrt{g(\mathbf{a})}$ ist. Aus Analogiegründen genügt es den ersten Fall weiter zu verfolgen. Da $g$ stetig ist, gibt es eine eventuell kleinere konzentrische $k$-dimensionale Kugel $B^{* *} \subset B^{*}$ derart, daß für alle $\mathbf{x} \in B^{* *} 0 \leq \sqrt{g(\mathbf{x})}<1$ 
ist. Sind $x_{1}, \ldots, x_{k}$ die Koordinaten eines Punktes $\mathbf{x}$ bezüglich obiger Basis, dann läßt sich für das Volumen von $\Psi\left(B^{* *}\right)$ folgende Abschätzung machen

$$
\begin{aligned}
\operatorname{Vol}_{k}\left(\Phi\left(B^{* *}\right)\right)=\operatorname{Vol}_{k}\left(\Psi\left(B^{* *}\right)\right) & =\int_{B^{* *}} \sqrt{g(\mathbf{x})} d x_{1} \wedge d x_{2} \wedge \ldots \wedge d x_{k} \\
& <\int_{B^{* *}} 1 d x_{1} \wedge d x_{2} \wedge \ldots \wedge d x_{k}=\operatorname{Vol}_{k}\left(B^{* *}\right) ;
\end{aligned}
$$

im Widerspruch zur Invarianz des $k$-dimensionalen Volumens.

Satz 3 Wenn bei einer einmal stetig differenzierbaren Abbildung $\Phi: \mathbb{R}^{n} \rightarrow \mathbb{R}^{n}$ alle $\Phi_{\mathbf{x}}^{\prime}$ $\left(\mathbf{x} \in \mathbb{R}^{n}\right)$ Isometrien sind, dann ist $\Phi$ eine Isometrie.

Beweis: Wir zeigen zunächst, daß die Bilder genügend kleiner Strecken kongruent zu ihren Originalen sind. Sei $\mathbf{a} \in \mathbb{R}^{n}$, dann ist $\Phi_{\mathbf{a}}^{\prime}$ eine Isometrie und $\Phi$ ist auf einer genügend kleinen Kugelumgebung $U(\mathbf{a})$ umkehrbar. Die lokale Umkehrabbildung ist dabei ebenfalls einmal stetig differenzierbar. Es gibt also eine Kugelumgebung $V(\Phi(\mathbf{a})) \subset \Phi(U(\mathbf{a}))$ und dazu wiederum eine in $U(\mathbf{a})$ gelegene Kugelumgebung $U^{*}(\mathbf{a})$ mit

$$
\Phi\left(U^{*}(\mathbf{a})\right) \subset V(\Phi(\mathbf{a})) .
$$

Wir wählen nun einen beliebigen Punkt $\mathbf{b} \in U^{*}(\mathbf{a})$ und berechnen die Länge des $\Phi$ Bildes der Strecke $S(\mathbf{a}, \mathbf{b}):=\{(1-t) \mathbf{a}+t \mathbf{b} \mid t \in[0,1]\}$. Da

$$
\Phi((1-t) \mathbf{a}+t \mathbf{b}), \quad t \in[0,1]
$$

eine einmal stetig differenzierbare Parameterdarstellung dieses Bildes ist, können wir seine Länge durch das übliche Integral darstellen und folgende Ungleichung anschreiben

$$
\begin{aligned}
\|\Phi(\mathbf{b})-\Phi(\mathbf{a})\| \leq \int_{0}^{1}\left\|\frac{d}{d t} \Phi((1-t) \mathbf{a}+t \mathbf{b})\right\| d t & =\int_{0}^{1}\left\|\Phi_{(1-t) \mathbf{a}+t \mathbf{b}}^{\prime}(\mathbf{b}-\mathbf{a})\right\| d t \\
& =\int_{0}^{1}\|\mathbf{b}-\mathbf{a}\| d t=\|\mathbf{b}-\mathbf{a}\| .
\end{aligned}
$$

Da $\Phi(\mathbf{a}), \Phi(\mathbf{b}) \in V(\Phi(\mathbf{a}))$ sind und $V(\Phi(\mathbf{a}))$ eine Kugel ist, liegt auch deren Verbindungsstrecke ganz in $V(\Phi(\mathbf{a}))$. Vermöge der Eigenschaften der lokalen Umkehrabbildung erhalten wir auf analoge Weise wie eben die folgende Ungleichung

$$
\|\mathbf{b}-\mathbf{a}\|=\left\|\Phi^{-1}(\Phi(\mathbf{b}))-\Phi^{-1}(\Phi(\mathbf{a}))\right\| \leq\|\Phi(\mathbf{b})-\Phi(\mathbf{a})\|,
$$

die uns den Schluß auf $\|\mathbf{b}-\mathbf{a}\|=\|\Phi(\mathbf{b})-\Phi(\mathbf{a})\|$ gestattet. Also besitzt jede Strecke mit Anfangspunkt a und Endpunkt $\mathbf{b} \in U^{*}(\mathbf{a})$ eine zu sich selbst kongruente Bildstrecke.

Nun zeigen wir, daß jede Strecke ein ihr kongruentes Bild besitzt. Sind $\mathbf{a}, \mathbf{b} \in \mathbb{R}^{n}$, dann ist die Verbindungsstrecke $S(\mathbf{a}, \mathbf{b})$ kompakt und daher Vereinigung von endlich vielen sukzessive überlappenden Strecken, deren Kleinheit garantiert, daß die Bildstrecken kongruent zu ihren Originalen sind. Daher ist auch das Bild von $S(\mathbf{a}, \mathbf{b})$ eine zu $S(\mathbf{a}, \mathbf{b})$ kongruente Strecke und somit ist $\Phi$ selbst eine Isometrie. 
Die Sätze 2 und 3 gestatten zuammengenommen folgende Formulierung.

Satz 4 Es sei $1 \leq k \leq n-1$. Ist $\Phi: \mathbb{R}^{n} \rightarrow \mathbb{R}^{n}$ eine $C^{1}$ - Abbildung, die das $k$ dimensionale Volumen aller $k$-dimensionalen Kugeln invariant läßt, dann ist $\Phi$ eine Isometrie.

Bemerkung: Damit ist das in [2] formulierte Problem von McMullen unter der zusätzlichen Annahme der stetigen Differenzierbarkeit gelöst. Ob man bei einem Homöomorphismus aus der bloßen Invarianz des $k$-dimensionalen Volumens im Sinne von Minkowski bereits auf die stetige Differenzierbarkeit schließen kann, weiß ich nicht.

Für die Kenntnis des Problems von McMullen und den Anreiz daran zu arbeiten danke ich Herrn Prof. F. Schnitzer von der Montanuniversität Leoben.

\section{Literatur}

[1] Wm. J. Firey: Affinities which preserve lower dimensional volumes. Am. Math. Monthly 72 (1965), p. 645.

[2] P. McMullen: Area Preserving Homeomorphisms. Elem. Math. 30 (1975), S. 86-87.

Peter Schöpf

Institut für Mathematik

Karl Franzens Universität

Heinrichstr. 36

A-8010 Graz

Österreich 